\title{
Announcing a New Affiliated Society Member of Blood Purification: The Japan Association for Clinical Engineers
}

The Japan Association for Clinical Engineers (JACE) is very pleased to announce its new status as an affiliated society member of Blood Purification, the official journal of the International Society of Blood Purification (ISBP), the Japanese Society of Hemodiafiltration (JS-HDF), and the Chinese Society of Blood Purification Administration (CSBPA). This exciting step forward for JACE offers our members a chance to learn more about the work of the broad range of professionals working globally in the field of blood purification and to participate in international discussions to advance the field. We hope too that readers can learn more about the roles and responsibilities of clinical engineers working in blood purification therapy in Japan.

Thanks to considerable advancements made in blood purification devices and technologies, therapies such as online hemodiafiltration (HDF) and intermittent infusion HDF (i-HDF) have been widely adopted in our countries. In Japan, clinical engineers have major roles and responsibilities in the safe implementation of these therapies, including being responsible for the purification of dialysis fluid to meet the standards set by the Japanese Society for Dialysis Therapy (JSDT) and for the maintenance and management of dialysis-related equipment.
As of 2017, the number of qualified clinical engineers stood at 41,533 in Japan. A 2013 survey by JACE showed that $94.6 \%$ of our members held a position either dedicated to or involved in blood purification therapy. Recent advancements in therapeutic technologies, such as performance improvement of dialyzers and water treatment equipment and the development of multifunctional automated dialysis machines, have made considerable contributions to improving patients' quality of life. Clinical engineers need to take a proactive approach to clinical research, including research on medical equipment management, for further improvement of patients' quality of life. We are proud of the indispensable role that clinical engineers play in blood purification therapy and in maintaining such high standards in dialysis treatment in Japan. Through our new affiliated society member status, I encourage all JACE members to contribute to facilitating communication and learning among the professionals involved in blood purification therapy worldwide.

\author{
Takashi Honma \\ Chief Director \\ Japan Association for Clinical Engineers
}

